\title{
Breathers on a Background: Periodic and Quasiperiodic Solutions of Extended Discrete Nonlinear Wave Systems
}

\author{
P.G. Kevrekidis* \\ Department of Mathematics and Statistics, University of Massachusetts, Lederle Graduate Research Tower, Amherst, MA \\ 01003-4515, USA \\ M.I. Weinstein \\ Mathematical Sciences Research, Bell Laboratories, Lucent Technologies, 600 Mountain Ave., Murray Hill, NJ 07974-0636
}

(October 30, 2018)

\begin{abstract}
In this paper we investigate the emergence of time-periodic and and time-quasiperiodic (sometimes infinitely long lived and sometimes very long lived or metastable) solutions of discrete nonlinear wave equations: discrete sine Gordon, discrete $\phi^{4}$ and discrete nonlinear Schrödinger. The solutions we consider are periodic oscillations on a kink or standing wave breather background. The origin of these oscillations is the presence of internal modes, associated with the static ground state. Some of these modes are associated with the breaking of translational invariance, in going from a spatially continuous to a spatially discrete system. Others are associated with discrete modes which bifurcate from the continuous spectrum. It is also possible that such modes exist in the continuum limit and persist in the discrete case. The regimes of existence, stability and metastability of states as the lattice spacing is varied are investigated analytically and numerically. A consequence of our analysis is a class of spatially localized, time quasiperiodic solutions of the discrete nonlinear Schrödinger equation. We demonstrate, however, that this class of quasiperiodic solution is rather special and that its natural generalizations yield only metastable quasiperiodic solutions.
\end{abstract}

\section{INTRODUCTION}

In the past two decades, there has been an explosion of interest in the study of solitary waves in spatially discrete nonlinear systems. Prominent examples, where one expects important phenomena to be intrinsically associated with spatial discreteness, are: the motion of dislocations in solid-state physics [1] 2], the transmission of kink-like bits of information in Josephson junction arrays [3], the propagation of pulses in nonlinear optical waveguides [4, 5] (where they present great potential for applications such as all-optical switching, steering and demultiplexing see e.g., [6] and references therein), or even in chemical [7] and biological 8 (and references therein) applications. Recent experimental work confirming the relevance of such models has appeared in [0,9,10].

The models arising in many of these studies, governing the behavior of a field $u_{n}(t)$ depending on a continuous time variable $t$ and discrete spatial variable $n$, are:

the discrete $S G$ equation (DSG)

$$
\ddot{u}_{n}=u_{n+1}+u_{n-1}-2 u_{n}-\frac{1}{d^{2}} \sin u_{n}
$$

the discrete $\phi^{4}$ equation $\left(D \phi^{4}\right)$

$$
\ddot{u}_{n}=u_{n+1}+u_{n-1}-2 u_{n}+\frac{1}{d^{2}}\left(u_{n}-u_{n}^{3}\right),
$$

and the discrete nonlinear Schrödinger equation (DNLS)

$$
i \dot{u}_{n}=-C\left(u_{n+1}+u_{n-1}-2 u_{n}\right)-\left|u_{n}\right|^{2} u_{n} .
$$

We have used the standard notation where $d=1 / \Delta x$ is the inverse spatial lattice spacing and $C=d^{2}=(\Delta x)^{-2}$ is the coupling constant.

An important phenomenon associated with spatial discreteness is the role of the spatial lattice sites as "defects" to which propagating states can be "trapped" or "pinned" 11 13]. At a structural level, this is a result of the breaking of translational invariance. Indeed, the spatially continuous variants are Lorentz invariant (SG, $\phi^{4}$ ) and Galilean invariant (NLS); a stationary coherent structure can be "boosted", i.e., transformed, into one traveling with a constant velocity.

The main coherent structures of interest in these equations (see i.e., 13 15, 19 21) are kink-like excitations in DSG and $D \phi^{4}$ and time periodic, exponentially localized in space pulse like excitations in DNLS (solitary waves). The latter are frequently referred to as discrete breathers.

In the analytical and numerical work on these discrete systems 11, 12, 19, 14. two main steady states have been

\footnotetext{
${ }^{*}$ To whom correspondence should be addressed:

Phone: 413-577-1977, fax: 413-545-1801, e-mail: kevrekid@math.umass.edu.
} 
identified; there are actually many other steady states [14,22. One is centered on a lattice site and one is centered between consecutive lattice sites. In the case of DSG and $D \phi^{4}$ kinks, the stable configurations (energy minimizers) are those centered between sites, while for the breathers of DNLS, stable configurations (constrained energy minimizers; the constraints are imposed by the additional conserved quantities such as the $l^{2}$ norm of the solution [18]) are those pulses which are centered at a lattice site.

Until recently, it was believed that an initial condition, which for the translation invariant systems leads to a coherent structure propagating to infinity at a uniform speed, would in these discrete systems result in a state radiating energy which would eventually get trapped by the "Peirels -Nabarro" potential [11] associated with the lattice and subsequently approach a time independent state. However, it was established in [13] that, depending on the parameter regime (lattice spacing), the asymptotic state may, in fact, be time-periodic. It may also consist of a time quasiperiodic state which slowly decays to a time-periodic state. The rate at which the system relaxes to its asymptotic state is controlled by resonances of "localized internal modes" with radiation modes. Internal modes are spatially localized modes associated with the linearized operator about the coherent structure with corresponding discrete eigenfrequencies (point spectrum), which are not related to an underlying group invariance (zero modes). They give rise to time periodic solutions of the linearized evolution equation, of the form $\phi_{n}^{\omega} \exp (i \omega t)$. Radiation modes are those associated with the continuous spectrum of the linearization.

In a recent paper [13], using a methodology developed in 16] (see also 17), we gave a systematic approach for deriving details of the decay to an asymptotic state. We now briefly describe these results. Suppose $\omega_{1}$ and $\omega_{2}$ are two internal mode frequencies associated with a coherent structure of (1), (2) or (3). Consider initial conditions which are a small perturbation of the coherent structure and that the perturbation (of $O(\varepsilon)$ ) consists of components in the direction of the internal modes, $\phi^{\omega_{1}}$ and $\phi^{\omega_{2}}$. As time evolves, nonlinearity generates various linear combinations of these frequencies so we investigate where such combinations land relative to the so-called phonon band (PB). Suppose that a combination of frequencies $k \omega_{1}+l \omega_{2}$ falls inside the PB with $|k|+|l|=n$. For DSG and $D \phi^{4}, \mathrm{~PB}$ is, respectively, the purely imaginary interval $\pm i\left[1 / d, \sqrt{4+1 / d^{2}}\right], \pm i\left[\sqrt{2} / d, \sqrt{4+2 / d^{2}}\right]$ and for DNLS it is $\pm i[\Lambda, \Lambda+4 C]$, where $\Lambda$ is the time-frequency of the ground state discrete solitary wave. In this case we showed in [13] that the time evolution of the internal mode powers (squared amplitudes), $P=P_{\omega_{1}}$ and $Q=Q_{\omega_{2}}$ is controlled by a system whose normal form is

$$
\begin{gathered}
P_{t}=-\sum_{k, l \in I^{\text {res }}} \varepsilon^{2(|k|+|l|-1)} \Gamma_{1 ; k l} P^{k} Q^{l} \\
Q_{t}=-\sum_{k, l \in I^{\text {res }}} \varepsilon^{2(|k|+|l|-1)} \Gamma_{2 ; k l} P^{k} Q^{l},
\end{gathered}
$$

where $I^{r e s}=\left\{k, l: k \omega_{1}+l \omega_{2} \in P B\right\}$ is the set of resonant frequency combinations. The "damping coefficients" $\Gamma_{1 ; k l}, \Gamma_{2 ; k l}$ can be computed [13], are always nonnegative and are generically strictly positive. From (4)(5), one concludes that the decay rate is controlled by the appropriate harmonic e.g., if $2 \omega_{1} \in \mathrm{PB}$, then the (long time asymptotic) decay of $P$ goes as $t^{-1 / 2}$, if $3 \omega_{1} \in \mathrm{PB}$, it goes as $t^{-1 / 4}$ and so on. As the parameters of the problem (such as the lattice spacing) vary, the set of resonances $I^{\text {res }}$ may vary and therefore so will the normal form equations and the nature of the asymptotic state dynamics; see [13 for examples of such transitions.

\section{ASYMPTOTIC STATES: STATIONARY AND PERIODIC, STABLE AND METASTABLE}

What are the possible asymptotic states ? Consider the discrete nonlinear wave equations (11) and (2). There is, of course, the ground state kink but one may ask whether there are additional time-periodic or even quasiperiodic states associated with the time-periodic internal modes., e.g., solutions of the nonlinear equation of the form: $K_{g s}+\sum_{\omega \in \Omega_{\text {internal }}} \phi_{n}^{\omega} \exp (i \omega t)+$ C.C. plus higher order corrections. Here, $K_{g s}$ denotes the ground state or least energy kink and $\Omega_{\text {internal }}$ denotes the set of localized internal mode frequencies. In [13] we prove the existence of time-periodic solutions (only one contribution to the above sum is present) in certain parameter regimes. No quasi-periodic solutions exist since for any internal mode frequencies $\omega_{1}, \omega_{2}$, there always exist integers $k_{1} \neq 0, k_{2} \neq 0$ such that $k_{1} \omega_{1}+k_{2} \omega_{2} \in \mathrm{PB}$. There are however metastable, very long-lived states of this type.

For DSG, depending on parameter regime, we have between one and three possible asymptotic states from among $K_{g s}$ (the ground state kink), $g W$ and $e W$, each with their own basin of attraction. $g W$ and $e W$ are time-periodic solutions associated with perturbed translational modes (i.e., bifurcating from the origin of the spectral plane) and edge (bifurcating from the edge of the continuous spectrum) internal modes (Theorem 5.1 of 13). See Table I.

For $D \phi^{4}$, we have between one and three from among $K_{g s}, g W, W$ and $e W$. The additional periodic solution $W$, is associated with the additional shape mode present in the point spectrum of the $\phi^{4}$ model. See Table II.

The above periodic solutions are localized timeperiodic excitations on a kink (spatially inhomogeneous) background, "breathers on kinks" (BOK's); see Fig. 1. Their dynamic stability in various regimes of the discreteness parameter $d$ was numerically investigated in [13]. 
We now turn to DNLS. Seek solutions of the form

$$
u_{n}(t)=\exp (i \Lambda t) \psi_{n} .
$$

This gives rise to a system of nonlinear algebraic equations for $\left\{\psi_{n}\right\}$

$$
-\Lambda \psi_{n}=-C\left(\psi_{n+1}+\psi_{n-1}-2 \psi_{n}\right)-\left|\psi_{n}\right|^{2} \psi_{n} .
$$

A variational approach to the questions of existence, excitation thresholds and nonlinear Lyapunov stability of the ground states of (7) is presented in [18]. Solutions can also be constructed by iterative methods implemented numerically in [20,21]. Linear stability analysis proceeds by seeking a solution of the form

$$
u_{n}(t)=\exp (i \Lambda t)\left(\psi_{n}+v_{n}(t)\right)
$$

where $v_{n}=a_{n} \exp (-i \omega t)+b_{n} \exp (i \omega t)$ denotes the perturbation about the ground state. This leads to the spectral problem

$$
\begin{gathered}
\omega a_{n}=-C \Delta_{2} a_{n}-2\left|\psi_{n}\right|^{2} a_{n}+\Lambda a_{n}-\psi_{n}{ }^{2} b_{n}^{\star}, \\
-\omega b_{n}=-C \Delta_{2} b_{n}-2\left|\psi_{n}\right|^{2} b_{n}+\Lambda b_{n}-\psi_{n}{ }^{2} a_{n}^{\star} .
\end{gathered}
$$

The analysis of the modes for different values of the relevant parameter $\Lambda / C$ is given in [19]. There it is found for the stable (site-centered) pulses of DNLS that there are two modes, a perturbed translational internal mode (the "pinning" mode) and an edge mode (the "breathing" mode). The picture for DNLS is derived in 19 and is analogous to that described above and in 13 for equations (11)-(2). In fig. 2, the relevant (localized) eigenmode frequencies are given and compared with those of the PB. The analysis of [19] shows that the methodology of 13. can be applied in this case, even though the basis of eigenfunctions is not orthonormal (the breathing mode has non-vanishing overlap with the rotational symmetry eigenmodes with $\omega=0$ ). However, a pseudo-scalar product can be used [19] in the spirit of [23] to perform the same analysis. Johansson and Aubry do this in two alternative ways (higher order perturbation theory and a conservation law approach) but nonetheless reach a special case of the result of 13] proving that when the $p$-th harmonic of a mode is inside $\mathrm{PB}$, then the amplitude of the mode decays as $|a(t)| \sim t^{-1 /(2 p-2)}$ asymptotically, as is suggested also by eqns. (4)-(5). However, in the DNLS case, they found, additionally, that the existence of the additional two-dimensional manifold of eigenmodes with zero frequency, in order to avoid unphysical divergences of higher order perturbations (due to projections on this manifold), causes an increase of the breather mode frequency according to $\Lambda_{t} \sim|a|^{2 p}$.

The methods of 13] can be used to extend the picture developed in [19]. Using the information, displayed in figure 2, on the location of linear combinations of internal mode frequencies associated with the ground state of equation (7), relative to the phonon band $\mathrm{PB}$, we can conclude:
- For any value of $\Lambda / C$ for which the edge mode exists $(0<\Lambda / C<1.7)$, initial conditions close to it decay as $t^{-1 / 2}$ since its second harmonic is always inside PB.

- The perturbed translational mode (which disappears around $\Lambda / C \sim 1.1$ as is noted in 19]), for $0.48<\Lambda / C<1.1,2 \omega_{g}$ is inside $\mathrm{PB}$, hence the decay in this range goes as $t^{-1 / 2}$. However, for $0.3997<\Lambda / C<0.48$, the third harmonic resonates with $\mathrm{PB}$ and hence the decay goes as $t^{-1 / 4}$. Subsequently $t^{-1 / 6}$ for $\Lambda / C \in[0.3576,0.3997]$ and $t^{-1 / 8}$ for $\Lambda / C \in[0.3304,0.3576]$ are implied by principle resonance occurring for higher harmonics.

Therefore, for any value of the coupling there are harmonics of the individual modes that are in the phonon band and hence cause decay of the internal mode oscillations. Hence, no genuinely periodic solutions along eigendirections can survive in this case (in the frame corotating with the discrete breather frequency $\Lambda$ ). The decay however can be extremely slow as we now explain. The perturbed translational mode, the neutral oscillatory mode to which the translation zero mode is perturbed for $\Delta x>0$, approaches zero very fast for small $\Lambda / C \sim \Delta x$, the continuum limit. This approach has been shown to be exponentially fast as $\Delta x \downarrow 0\left(\omega^{2} \sim \exp \left(-\pi^{2} / \Delta x\right)\right)$ in [24,25]. Therefore, as seen in figure 2 and can be derived from the above estimates, for very small values of $\Lambda / C$, only very high harmonics will fall inside $\mathrm{PB}$. By the analysis of 13] this corresponds to very slow time decay rates of the internal mode oscillations, which may be hard to detect numerically. An example of this sort is given in figure 3. The numerical experiment is performed for DNLS, with $\Lambda / C=0.370$ and for the perturbed translational mode of $\omega / C=0.102$, the 4-th harmonic is the leading one in the phonon band. The initial condition is taken to be the exact breather profile with a small perturbation along the perturbed translational mode eigendirection. The background oscillations are clearly discernible as time-dependent side wobbles in the evolving profile. Our simulations show very slow decay of these oscillations, in accordance with the theoretical prediction. The analysis of 13] implies that these modes will decay to the ground state standing wave breather but only over very long time scales. Modes such as the one that is observed in figure 3 are very long-lived time-periodic and spatially localized. In view of (8) they correspond to slowly decaying (metastable) quasi-periodic oscillations of DNLS (which are periodic in the rotating frame). The periodic oscillation is mounted on a breather background. Hence these modes are (metastable) Breathers On Breathers (BOB's). We note that there are other constructions of quasiperiodic modes in discrete systems. Such attempts 
(see, for example, 26]) exploited continuation from the anti-continuum limit of two oscillators with different frequencies. Also, for polaron type models such as the Holstein model, a proof of existence of quasiperiodic solutions was give in 22. Our analysis above is not restricted to the regime where the sites are weakly coupled. However, it should be noted that very close to the continuum limit, logarithmic effects similar to the ones described in 27] for generalized continuum NLS equations may become relevant. The work presented herein mainly focuses in the regimes of large or moderate discreteness and the mechanisms described will still be present even for very small discreteness. The work of [27, however, raises the interesting question as to whether there is a crossover point beyond which (in approaching the continuum limit) logarithmic effects become dominant over power law ones. Such issues will be addressed in a future publication.

\section{INFINITELY LONG-LIVED QUASIPERIODIC SOLUTIONS}

Are there time quasi-periodic states which are nondecaying? We now display a family of (non-decaying) time quasi-periodic and spatially localized BOB states. Our point of departure will be the modes introduced in [6], which we call twisted localized modes (TLM's). These modes are solutions of DNLS of the form (6) with $\left\{\psi_{n}\right\}=(\ldots, \alpha, 1,-1,-\alpha, \ldots)$ with $0<\alpha<<1$. They can be viewed as soliton-antisoliton bound pairs which can exist due to discreteness 28,29]. There is no continuum analog since in the continuum case a solitonantisoliton initial condition naturally repels.

Henceforth, for simplicity we will fix $\Lambda=1$ and vary $C$ only. We construct these modes numerically by solving equation (7) and subsequently solve numerically eqs. (9)-(10) to determine their linear stability. We find that

- TLM's are stable for $1 / C \leq 0.146$. Their spectrum for this parameter range consists of two modes at $\omega=0$ and an additional internal mode with frequency $\omega_{\star}$ in the gap between 0 and edge of PB.

- For values $1 / C>0.146$ a series of instabilities arise giving quite interesting phenomena that have been analyzed elsewhere 29].

For $C$ in the range of stability, the location of multiples of the single non-zero frequency $\left(\omega_{\star}\right)$ relative to $\mathrm{PB}$ is displayed in figure 4 . It can be seen that

- For $1 / C \in[59.712,67.681], 4 \omega_{m} \in \mathrm{PB}$ and hence implying decay of the internal mode oscillation about the TLM with a rate: $t^{-1 / 6}$. For $1 / C \in$ [31.943, 39.604], $3 \omega_{m} \in \mathrm{PB}$ implying $t^{-1 / 4}$ decay and for $1 / C \in[11.989,19.288], 2 \omega_{m} \in \mathrm{PB}$ implying a $t^{-1 / 2}$ decay rate.
- More interestingly, as figure 4 suggests, the gaps between the above coupling parameter intervals constitute parameter regimes for which no multiples of $\omega_{\star}$ lie inside PB. Hence an initial condition consisting of the exact mode plus a perturbation along the $\omega_{\star}$ eigendirection encounters no evident decay mechanism. Indeed, the proof of Theorem 5.1 of 113 can be carried through in this case to obtain the existence of time-periodic solutions of the nonlinear perturbation equation governing $v_{n}(t)$. In this way, we have constructed genuine time quasiperiodic solutions (BOB's) of DNLS of the form (8), where $v_{n}(t)$ is time periodic. This is demonstrated in figure 5, where we display the results of a numerical experiment for $C=0.02, \Lambda=1$, where the internal mode frequency is $\omega_{\star}=0.294$. We observe the very regular oscillations on top of the breather amplitude due to the BOB's quasi-periodicity.

\section{GENERALIZATION TO MULTIPULSES}

We saw in the previous section that TLM's can be thought of as a pulse and an antipulse whose "backs" have merged. The question that then stems from this analogy is whether genuinely quasiperiodic solutions of DNLS arise naturally in such multipulse setups where two or more pulses have been concatenated.

A general analysis of such configurations for discrete setups has been given in 28] for two pulses and generalized in 30] for multiple pulses. In these works it has been found that in order for multipulses to be stable, the nearest neighbor ones have to possess opposite parity, i.e., a phase difference of $\pi$. Hence, we will consider only such configurations hereafter.

In order to understand the possibilities for quasiperiodic solutions of multipulse type, let us return to the linearization picture for a single pulse. As is well known, for the single pulse, there are two pairs of eigenvalues near the origin of the spectral plane, the rotational modes (of zero frequency) and the translational modes of frequency dependence (on $h)$ as $\exp \left(-\pi^{2} / h\right)$. For a concatenation of $\mathrm{N}$ pulses, there will be $2 N$ pairs near the origin. $N$ of them will be translational (stemming from the linear combinations of the translational eigenmodes of the individual pulses), 1 pair will be the rotational one of $\omega=0$ and $N-1$ pairs will pertain to the so-called interaction eigenmodes 28,30], whose frequencies will, in general, behave as $\exp (-L)$, where $L$ is the (typical) pulse separation. It should be noted that for such multipulse configurations to occur, it necessary for the pulses to have a separation larger than a minimal one $L>L_{m i n}$. 
For values of $h \leq O(1)$, as was found in 28, 30, it is generically true that for $L>L_{m i n}$, the interaction eigenvalues have frequencies typically lower than the translational ones. Hence, since as we saw in section II, quasiperiodic solutions cannot be formed for such regimes of the lattice spacing, as the translational eigenvalues resonate with the continuous spectrum, hence such solutions will also not be but metastable for the multipulse configurations as well.

From the above argument, it can be seen that for multipulse configurations, as was the case with the TLM's of section III, only strong discreteness contexts $h>>1$ provide the possibility for quasiperiodic solutions. In such regimes of strong discreteness, the translational eigenmodes have already merged with the continuous spectrum 19] and only the interaction eigenmodes (and their harmonics) relative position to the continuous spectrum has to be accounted for.

In order to understand the behavior of multipulses, a natural continuation parameter that we will use in this context is the separation between the pulses $L$. In the case of the TLM's, this separation will be considered $h$, i.e., the distance between the pulse maximum and the antipulse minimum. In this case, the form of the solution is shown in the top left panel of Fig. 6 and the form of the evolution of $\omega / C$ as a function of $\Lambda / C$ is given in the top right panel. In the middle two and lower two panels of fig. 6, similar results are shown for $L=2 h$, and $L=3 h$ respectively. What can be clearly observed is that in these cases, the dependence of the internal (interaction) eigenmode on the coupling constant is such that for any value of $h$, there are harmonics of the internal mode frequency inside the PB. Hence, the TLM's are very special in the sense that the relation of their centers positions is such that it permits for a set of intervals of coupling constants to have no resonances of internal modes with the PB and, thus, quasiperiodic solutions.

To show the generality of the above conclusion, we performed similar numerical experiments for 3-pulse configurations (in which the middle one was of opposite phase than the two outer ones). The case of $L=h$ is once again shown in the top left panel of Fig. 7 and the corresponding resonance diagram of $\omega / C$ as a function of $\Lambda / C$ appears in the top right panel of the same figure. The same plots for $L=2 h$ and $L=3 h$ are shown in the middle and lower rows of Fig. 7 respectively. In this case, as per the general discussion presented above, there are two pairs of interaction eigenmodes. In the case of $L=h$, it can be observed that their frequency increases as a function of $\Lambda / C$ and hence gaps appear in the presence of the modes' harmonics in the PB. Such gaps for instance are found for $h \in(4.677,5.292), h \in(6.537,7.482)$ for the first frequency (in solid line) or for $h \in(4.321,6.468)$ for the second internal mode (in dashed line). Excitation of one of these internal modes in the respective intervals will give rise to genuinely quasiperiodic solutions.
On the other hand, it can be observed that already for $L=2 h$, once again the dependence of $\omega / C$ on $\Lambda / C$ is monotinically decreasing and that harmonics will always be present inside. The same picture of course in maintained for $L=3 h$ and for larger interpulse distances.

These results lead us to the conclusion that generically quasiperiodic solutions of the TLM variety (and its multipulse generalization) will be present in DNLS type systems. However, this is a rather "delicate" result of the interplay between the lattice spacing $h$ and the interpulse separation $L$ which has been found to be quite special to such. More general multipulse solutions of larger interpulse distance are invariably found to possess internal modes whose harmonics are resonant with the PB and hence in the latter case only metastable quasiperiodic configurations will be present.

\section{CONCLUSIONS}

In this paper, we have given theoretical justification as well as numerical evidence of the existence and asymptotic stability of breather-like modes which consist of (depending on parameter regime) infinitely long lived or very long lived spatially localized and time-periodic oscillations on a kink background (BOK's) or on a breather background (BOB's). These modes are different from previously reported ones in that they reside on a nonuniform (coherent structure) background rather than on a uniform background. Because of their relevance to asymptotic states for a wide range of initial conditions (possibly in experimental situations of interest) further study and understanding of these modes as well as analytical investigation of their stability are challenging questions worth pursuing. The existence of genuinely quasi-periodic solutions is apparently rare for nonlinear conservative wave equations on infinite spatial domains. This is in contrast to the case of nonlinear wave equations on finite domains. The discrete case corresponds to finite dimensional KAM theory. The continuum case (that of vibrations, strings and membranes) is studied in 31 33, where such solutions have been constructed. The non-generic nature of such quasi-periodic solutions was however demonstrated for discrete systems in the infinite domain, and their generalization to multipulse type configurations was also considered.

[1] J. Frenkel, T. Kontorova J. Phys. USSR , 1137 (1939).

[2] W. Atkinson, N. Cabrera Phys. Rev. A, 138, 763 (1965).

[3] A.V. Ustinov, T. Doderer, I.V. Vernik, N.F. Pedersen, R.P Huebener, V. A. Oboznov Physica D, 68, 41 (1994). 
[4] J. Kutz, C. Hile, W. Kath, R.-D. Li, P. Kumar J. Opt. Soc. Am. B., 11, 2112 (1994).

[5] J. Kutz, C. Hile, W. Kath, R.-D. Li, P. Kumar Opt. Lett., 18, 802 (1993).

[6] S. Darmanyan, A. Kobyakov and F. Lederer, Sov. Phys. JETP, 86, 682 (1998).

[7] B. L. Swanson et al. Phys. Rev. Lett., 82, 3288 (1999).

[8] M. Peyrard and A.R. Bishop, Phys. Rev. Lett., 62, 2755 (1989).

[9] A. Campa and A. Giasanti physics/9802043, preprint (1998).

[10] H.S. Eisenberg, Y. Silberberg, R. Morandotti, A.R. Boyd \& J.S. Aitchison, Phys. Rev. Lett. 81, 3383 (1998).

[11] M. Peyrard and M.D. Kruskal, Physica D, 14, 88 (1984).

[12] J.A. Combs and S. Yip, Phys. Rev. B, 28, 6873 (1983).

[13] P.G. Kevrekidis and M.I. Weinstein, Physica D, 142, 113 (2000).

[14] N.J. Balmforth, R.V. Craster and P.G. Kevrekidis, Physica D, 135, 212 (2000).

[15] P.G. Kevrekidis and C.K.R.T. Jones, Phys. Rev. E, 61, 3114 (2000).

[16] A. Soffer and M.I. Weinstein, Invent. math. 136, 9-74 (1999)

[17] D. Pelinovsky, Yu.S. Kivshar and V.V. Afanasjev, Physica 116D, 121 (1998).

[18] M.I. Weinstein, Nonlinearity, 12, 673 (1999).

[19] M. Johansson and S. Aubry, Phys. Rev. E, 61, 5864
(2000).

[20] P.G. Kevrekidis, K.Ø. Rasmussen and A.R. Bishop, Phys. Rev. E, 61, 2006 (2000).

[21] P.G. Kevrekidis, K.Ø. Rasmussen and A.R. Bishop, Phys. Rev. E, 61, 4652 (2000).

[22] S. Aubry Physica D, 103, 201 (1997)

[23] D.J. Kaup, Phys. Rev. A, 42, 5689 (1990).

[24] P.G. Kevrekidis, T. Kapitula and C.K.R.T. Jones, Phys. Lett. A, 269, 120 (2000).

[25] T. Kapitula and P.G. Kevrekidis Nonlinearity, 14, 533 (2001).

[26] M. Johansson and S. Aubry, Nonlinearity, 10, 1151 (1997).

[27] D.E. Pelinovsky, Physica 119D, 301 (1998).

[28] T. Kapitula, P.G. Kevrekidis and B.A. Malomed Phys. Rev. E, 63, 036604 (2001).

[29] P.G. Kevrekidis, A.R. Bishop and K.Ø. Rasmussen, Phys. Rev. E, 63, 036603 (2001).

[30] P.G. Kevrekidis, Multipulses in Discrete Hamiltonian Nonlinear Systems (submitted).

[31] W. Craig, C.E. Wayne, Commun. Pure Appl. Math. 46, 1409-1498 (1993)

[32] S. Kuksin, Lecture Notes in Math. 1556 Springer Verlag, Berlin Heidelberg New York, 1993

[33] J. Bourgain, Lecture Notes in Pure and Appl. Math. 186, Dekker, New York 1997

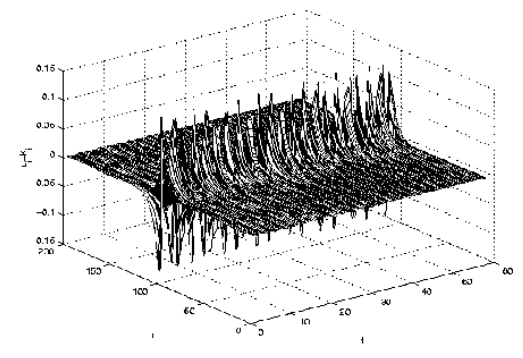

FIG. 1. BOK background: Discrete SG simulation result for time evolution of an initial condition consisting of a kink plus a projection to the edge mode. Shown is the time evolution of the initial condition when substracting $K_{g s}$ from the profile. The persistent oscillations of non-decreasing amplitude indicate that the BOK is indeed the asymptotic state. 


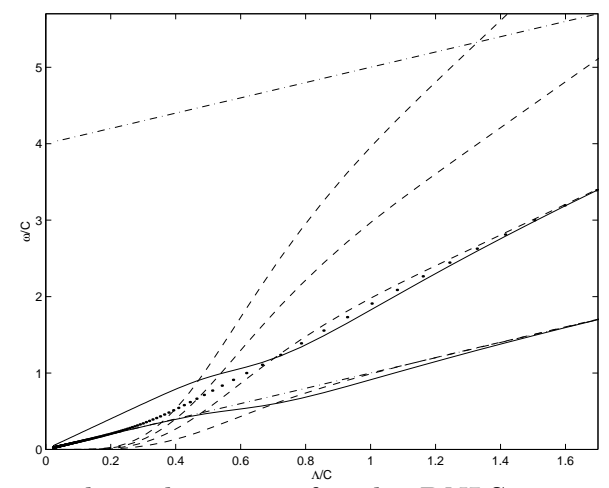

FIG. 2. Internal Eigemodes, harmonics and combinations for the DNLS case: The translational mode and its first few harmonics are shown in dashed line, the edge mode and its second harmonic shown in solid line, the sum of the two frequencies is given by the dotted line. The band edges of the PB are shown by dash-dot lines.
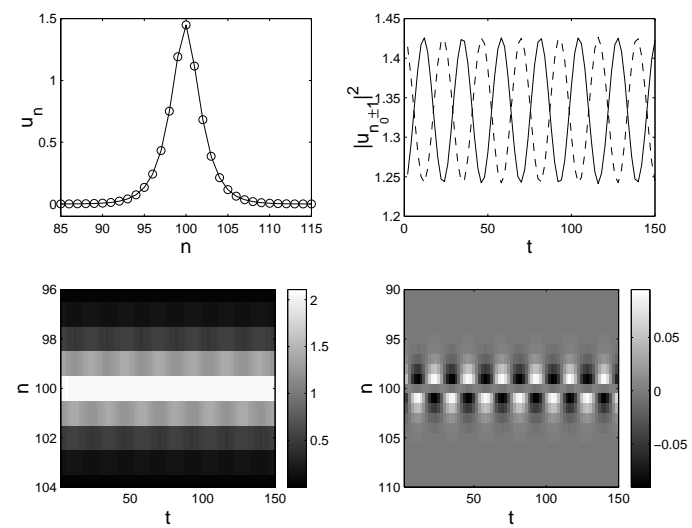

FIG. 3. Example of a metastable BOB: For the DNLS and $\Lambda / C=0.370$ the time evolution of a BOB is shown. The top left panel shows the initial condition consisting of the exact breather plus a perturbation along the direction of the eigenvector of the translational eigenmode. The top right panel shows the time evolution of the amplitude squared of the adjacent to the central ( $n_{0}=100$ in this case) lattice sites clearly indicating the persistence of oscillations on top of the original breather. The bottom left panel shows the contour plot of the spatio-temporal evolution of the full BOB while the bottom right shows the spatio-temporal evolution of the (amplitude squared) of the BOB minus the exact breather. This also clearly indicates the persistence of the metastable BOB (notice that very slow decay is observed for the simulated times).

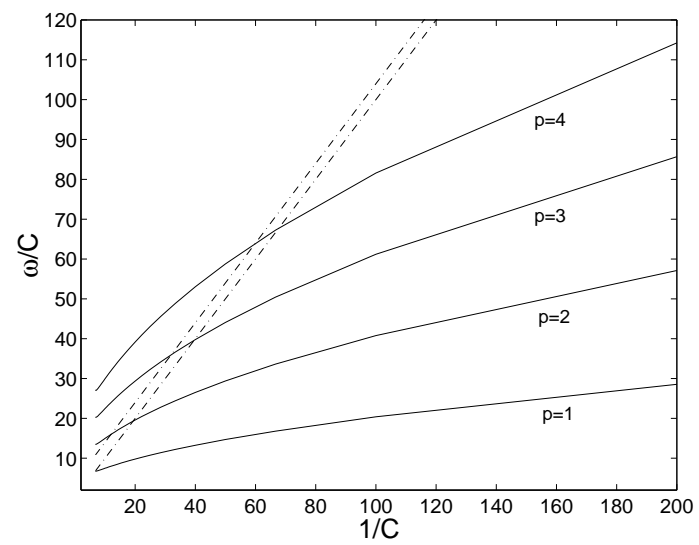

FIG. 4. Relative position of the internal mode frequency $\omega_{\star}$ of the DKL solution and its $p-t h$ harmonics (solid lines) with respect to $\mathrm{PB}$ (dash-dotted lines) for different values of the coupling constant $C . \Lambda=1$. 


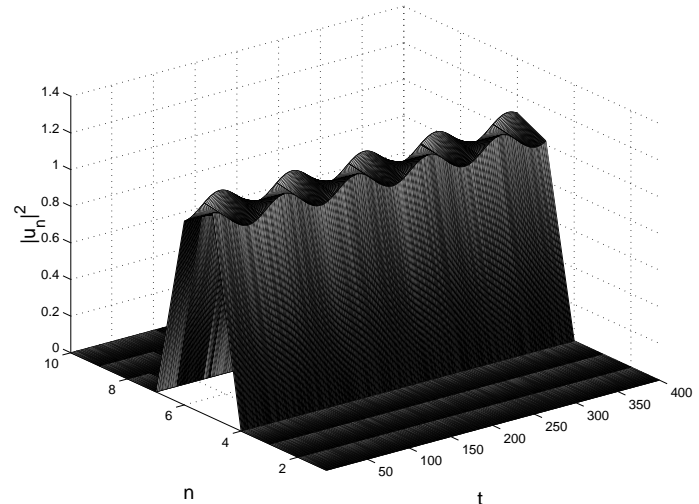

FIG. 5. Proof of persistence of DKL BOB mode for $C=0.02$. The additional frequency of the oscillations clearly observed at the top is checked to agree with the predictions of linear stability theory for $\omega_{\star}$. Shown is the spatio-temporal evolution of the amplitude squared $\left(\left|u_{n}(t)\right|^{2}\right)$ of the field (see eqn. (8)). 

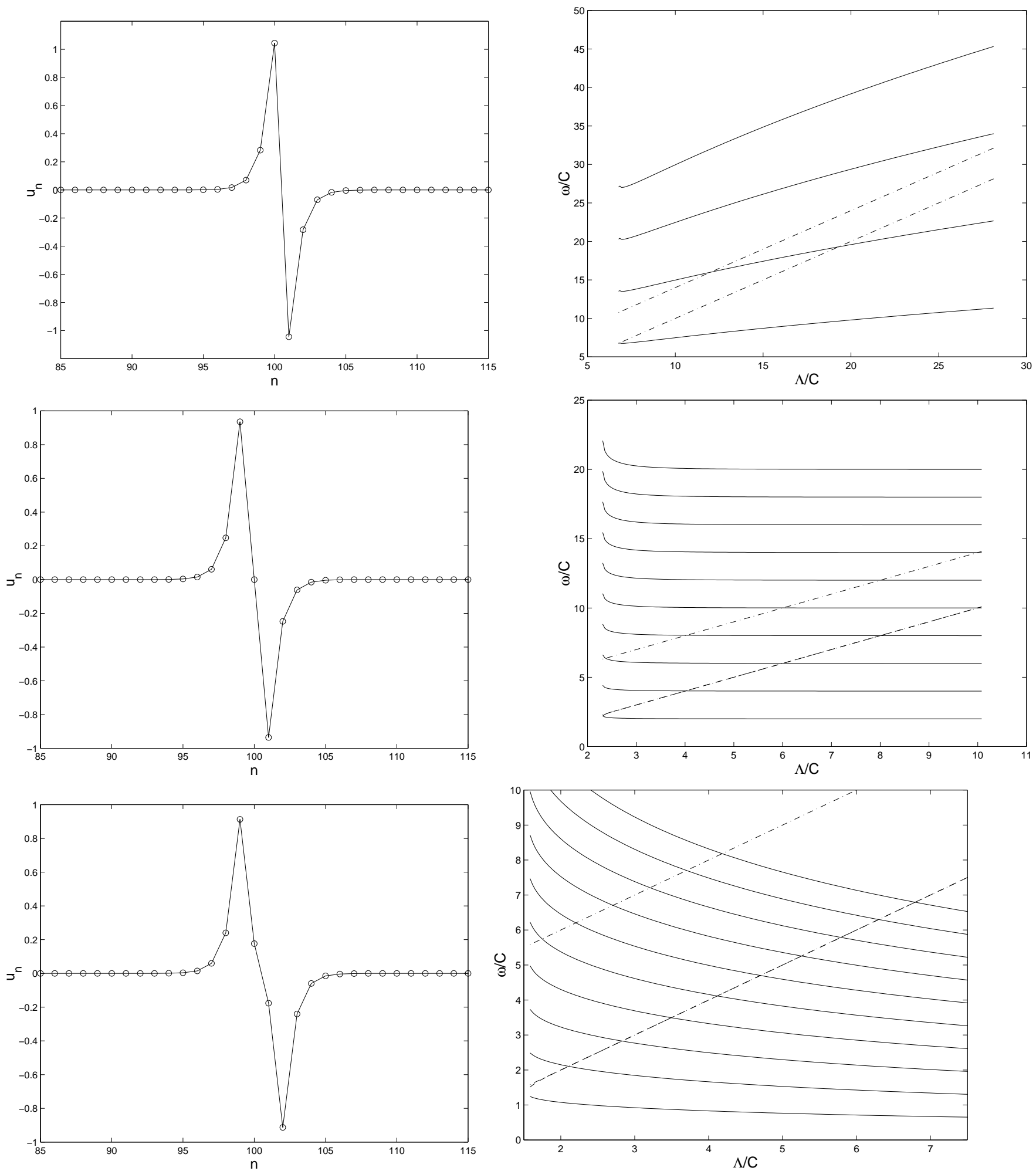

FIG. 6. The generalization of the twisted modes and their respective resonance pictures. The top left panel shows the spatial profile of the TLM and the top right, the frequency its internal mode and its first few harmonics (given by solid line) with the PB (the edges of which are given by dash-dotted lines. The middle row shows the extension of the TLM for distance between centers equal to $L=2 h$ (see also text); once again the left panel shows the spatial profile, while the right shows the internal frequency and its harmonics positions with respect to PB. The same plots are shown in the bottom row for a TLM with $L=3 h$. It is clear that in both the middle and bottom right panels the decreasing dependence of the interaction mode frequency (divided by $C$ ) as a function of the inverse coupling constant generically allows for the occurrence of resonances and hence BOB type solutions can only be metastable. $\Lambda=0.5$. 

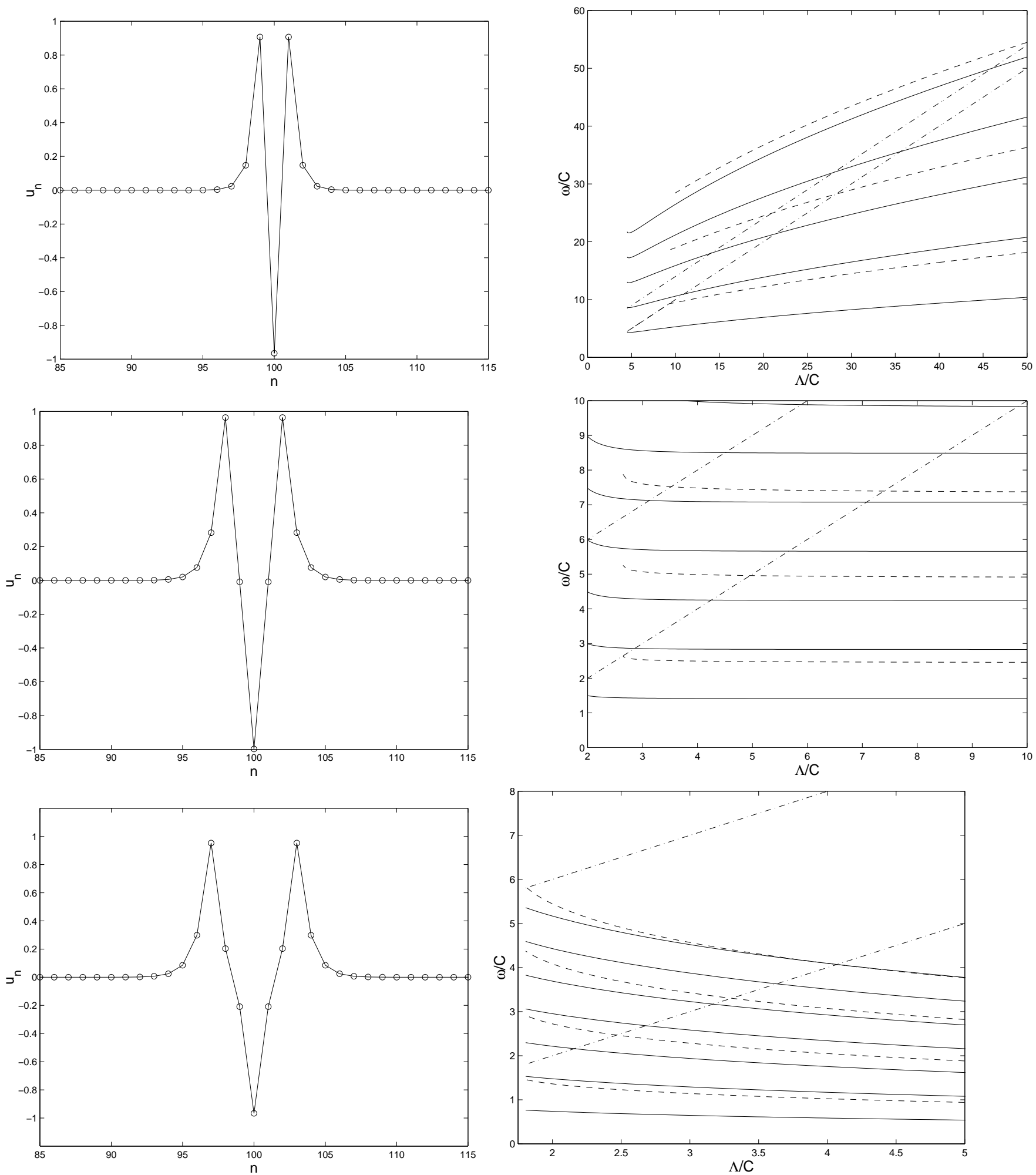

FIG. 7. Similar plots as the ones of Fig. 6 are given here for the generalization to three pulses. The mode with $L=h$ is given in the top left panel while the resonance picture of its two internal (interaction) eigenmodes (given by solid and dashed line respectively) and their harmonics with respect to to the PB (lying between the two dash-dotted lines) is given in the top right panel. Similar plots for the $L=2 h$ three and the $L=3 h$ three pulse solutions are given in the middle and bottom row. Notice once again that only for the $L=h$ case there is an increasing dependence of $\omega / C$ as a function of $\Lambda / C$ for the internal modes and hence there is a possibility for genuinely quasiperiodic solutions. As discussed also in the text for $L \geq 2 h$, the decreasing nature of the above dependence generates resonances with the PB and hence becomes prohibitive for the presence of (other than metastable) such solutions. 
TABLE I. SG normal form, equilibria and anticipated coherent structures

\begin{tabular}{|l|l|l}
\hline \hline Regime of $d$ & Equilibria & Coherent structures \\
\hline I: $d<d_{e}, d_{e} \sim 0.515$ & $P \geq 0$ & $K_{g s}, g W$ \\
\hline II: $d_{e} \leq d<0.565$ & $\{(P, 0),(0, Q): P \geq 0, Q \geq 0\}$ & $K_{g s}, W, g W$ \\
\hline III: $0.565 \leq d<d_{*}$ & $\{(0, Q): Q \geq 0\}$ & $K_{g s}, W$ \\
$d_{*} \sim 0.86$ & & $K_{g s}$ \\
\hline IV $: d_{*} \leq d$ & $\{(0,0)\}$ & \\
\hline \hline
\end{tabular}

TABLE II. $\phi^{4}$ normal form, equilibria and anticipated coherent structures

\begin{tabular}{|l|r|r}
\hline \hline Regime of $d$ & Equilibria & Coherent structures \\
\hline I: $d<0.5398$ & $\{(P, 0): P \geq 0\}$ & $K_{g s}, W, g W$ \\
& $\{(0, Q): Q \geq 0\}$ & $K_{g s}, W$ \\
\hline II-III: $0.5398 \leq d<0.6364$ & $\{(0, Q): Q \geq 0\}$ & $K_{g s}, W, g W$ \\
\hline IV: $0.6364 \leq d<0.6679$ & $\{(0, Q): Q \geq 0\}$ & $K_{g s}, W$ \\
& $\{(P, 0): P \geq 0\}$ & \\
\hline V: $0.6679 \leq d<d_{e}$ & $\{(0, Q): Q \geq 0\}$ & $K_{g s}, W, e W$ \\
$d_{e} \sim 0.82$ & $\{(0, Q, 0): Q \geq 0\}$ & \\
\hline VI: $d_{e} \leq d<0.9229$ & $\{(0,0, R): R \geq 0\}$ & $K_{g s}, e W$ \\
\hline VII: $0.9229 \leq d<1.2234$ & $\{(0,0, R): R \geq 0\}$ & \\
$d_{*} \sim 1.2234$ & $\{(0,0,0)\}$ & $K_{g s}$ \\
\hline VIII: $d_{*} \leq d$ & & \\
\hline \hline
\end{tabular}




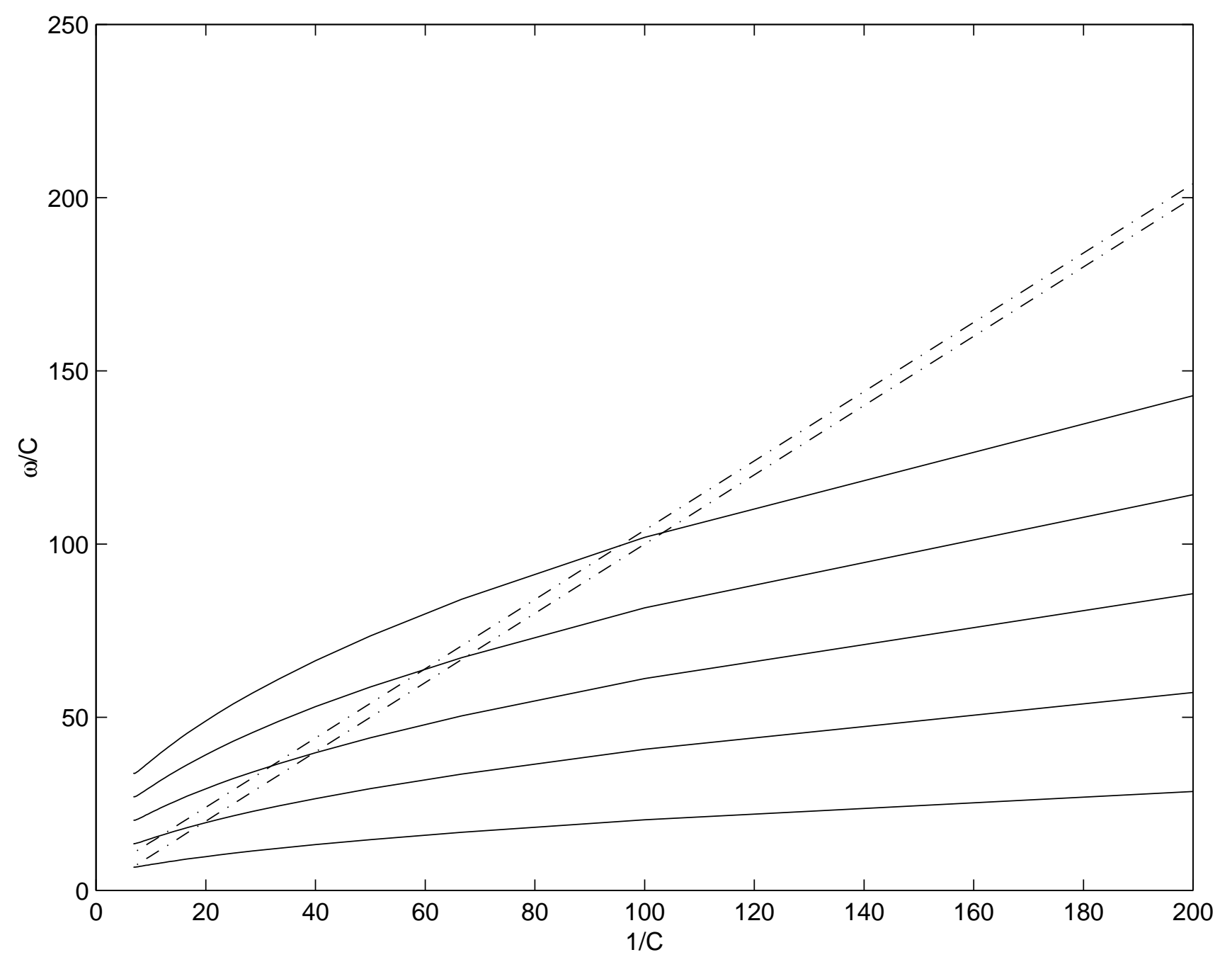




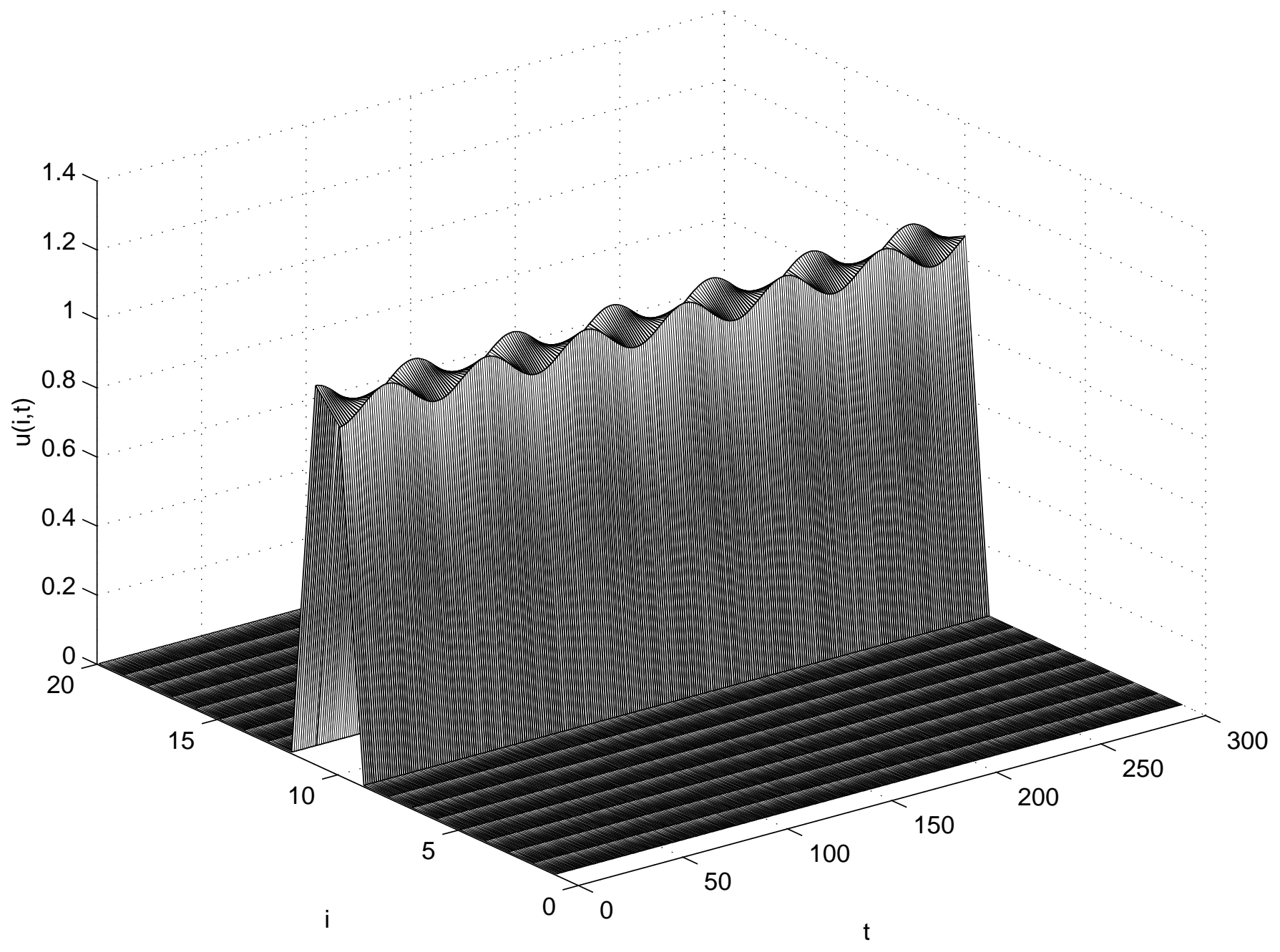

